creases more in response to a standardised cold stimulus than control divers with no problems diving in cold water.

There are no long term complications of this condition. A group of 11 divers who had had documented pulmonary oedema on immersion in cold water was followed for a mean of eight years. All had above average exercise tolerance. Seven went on to develop mild hypertension and one developed atrial fibrillation. There were no deaths, and no one developed symptomatic heart disease.

\section{CONCLUSION}

The development of pulmonary oedema on immersion in cold water has obvious repercussions for the recreational diver. With the increased popularity in diving it is important that staff in accident and emergency departments are aware of this condition. Treatment is easy and is symptomatic only. There do not appear to be any long term serious complications, except that a watch should be kept for the development of hypertension in the ensuing years and the patient should be encouraged to develop other leisure interests.

1 Wilmhurst PT, Nuri M, Crowther A, Webb-Peploe MM. Cold-induced pulmonary oedema in scuba divers and swimmers and subsequent development of hypertension. Lancet 1989;i:62-5.

2 Edmonds C, Lowry C, Pennefather J. Diving and subaquatic medicine, 3rd ed. London: Butterworth Heinemann, 1994 medicine, 3rd ed. London: Butterworth Heinemann, 1994.
Bennett PB, Elliott DH. The physiology of medicine and diving, 4th ed. Philadelphia: W B Saunders, 1993.

\title{
Silent myocardial infarction during hypoglycaemic coma
}

\author{
F M Saunders, T Llewellyn
}

\begin{abstract}
A case of silent myocardial infarction associated with hypoglycaemic coma is described. A hypoglycaemic episode represents a risk factor for a patient with underlying ischaemic heart disease. A routine ECG may be indicated in such circumstances.

$(\mathcal{A}$ Accid Emerg Med 1996;13:357-358)
\end{abstract}

Key terms: hypoglycaemia; diabetes mellitus; myocardial infarction

\section{Case report}

A 75 year old woman with ischaemic heart disease and non-insulin-dependent diabetes mellitus controlled by metformin presented to the emergency department following a period of unconsciousness at home. The patient had been found by her daughter and was described as being cold, clammy, and unrousable. An ambulance was called, and on arrival at the scene the crew made a provisional diagnosis of hypoglycaemia and put jam and sugar into the unconscious patient's mouth. Within minutes she had regained consciousness and was talking to her rescuers.

On arrival at the emergency department the patient was fully conscious and alert, with no history of chest pain either before or after her period of unconsciousness. Clinical observations were normal and a Glucostix test gave a reading of $16.4 \mathrm{mmol} /$ litre. A routine ECG was requested by the nursing staff and this revealed evidence of an acute inferior myocardial infarction, with $5 \mathrm{~mm}$ of ST elevation in leads II, III, and aVF and T wave inversion in III and aVF. The patient was immediately transferred to the resuscitation area and the medical staff informed.

Clinical examination revealed a raised jugular venous pressure, mild ankle oedema, and severe diabetic retinopathy, but was otherwise unremarkable. Formal laboratory blood glucose estimation confirmed the reading of 16.4 $\mathrm{mmol} /$ litre.

A diagnosis of silent myocardial infarction was made and the patient was admitted to an acute medical ward. Serial ECGs and serum aspartate transaminase and creatine kinase estimations over the next 72 hours confirmed the diagnosis. She had an uneventful hospital stay and was discharged home seven days later.

\section{Discussion}

It is recognised that hypoglycaemia increases damage to the ischaemic myocardium. ${ }^{1}$ However, few cases of myocardial infarction associated with hypoglycaemia have previously been reported. ${ }^{23}$ Others have described ischaemic ECG changes during severe hypoglycaemia which resolved completely after treatment with intravenous glucose. ${ }^{4}$ The development of hypoglycaemia was not preceded by chest pain in any of these cases. It has been shown that autonomic neuropathy in diabetes mellitus leads to disturbed cardiac perception and thus may play a role in silent myocardial infarction. ${ }^{5}$

Myocardial infarction normally tends to increase the blood sugar because of a decrease in insulin sensitivity ${ }^{6}$; therefore, had it been the primary event in our patient, symptomatic hypoglycaemia would have been less likely. Although we have no biochemical proof that 
the period of unconsciousness was due to hypoglycaemia the history and rapid response to buccal glucose allows a confidant clinical diagnosis to be made. According to Libby et al there are many possible ways in which hypoglycaemia could be deleterious to the myocardium. Severely ischaemic myocardium is dependent on anaerobic pathways for its energy production and is thus uniquely dependent on carbohydrate substrates. In addition, hypoglycaemia is known to produce a sympathoadrenal discharge which could increase heart rate, ventricular afterload, and the inotropic state of the myocardium, with a resulting increase in myocardial oxygen consumption.

In spite of the paucity of recorded cases, there seems to be evidence that a hypoglycae- mic episode presents a period of risk for myocardial infarction, particularly in patients with underlying ischaemic heart disease. A routine ECG in such circumstances may reveal more cases.

1 Libby P, Maroko PR, Braunwald E. The effect of hypoglycaemia on myocardial ischaemic injury during acute experimental coronary artery occlusion. Circulation 1975;51:621-6.

2 Arora RR, Meisheri YV, Joshi V. Myocardial infarction secondary to hypoglycaemia. Case reports. Indian Heart $\mathcal{F}$ 1980;32:186-9.

3 Wray R, Sutcliffe SB. Propranolol-induced hypoglycaemia and myocardial infarction. BMF 1972;ii:592.

4 Markel A, Keidar S, Yasin K. Hypoglycaemia-induced ischaemic ECG changes. Presse Med 1994;23:78-9.

5 Pauli P, Hartl L, Marquardt C, Stalmann H, Strian F. Heartbeat and arrhythmia perception in diabetic autoHeartbeat and arrhythmia perception in diabe

6 Oganov RG, Aleksandrov AA, Vinogradova IV. Sensitivity to insulin in acute myocardial infarction. Kardiologiia 1977; 17:89-94.

\title{
Inadvertent subclavian artery puncture in an intravenous drug abuser
}

\author{
Gordon W McNaughton, Ian J Swann
}

\begin{abstract}
Arterial complications from inadvertent arterial puncture in intravenous drug abusers are well recognised. Most reports describe injury to the femoral artery with occasional reports of brachial and radial artery injury. A case of subclavian artery injury is described in this paper, and the pathophysiology and treatment of intraarterial drug injection discussed.

( $F$ Accid Emerg Med 1996;13:358-359)
\end{abstract}

Key terms: subclavian artery injury; intravenous drug abuse

\section{Case report}

A 25 year old male intravenous drug abuser presented to the accident and emergency (A\&E) department 30 minutes after injecting heroin into his right supraclavicular fossa. He experienced transient pain and paraesthesiae from the injection site down to his hand. This was followed by severe pain and erythema in a well demarcated area over the right side of his neck and supraclavicular fossa. The skin was warm, erythematous, and hypersensitive. Distal pulses were normal but a bruit was audible in both the supraclavicular fossa and in the axilla. The patient was admitted to hospital for observation. His symptoms and signs resolved over a three hour period and he was discharged later that day. He reattended the A\&E department 10 days later with a heroin overdose. Further assessment at that stage confirmed complete resolution without residual bruit.

\section{Discussion}

Glasgow is comparable to many other inner city areas in that it has a large population of

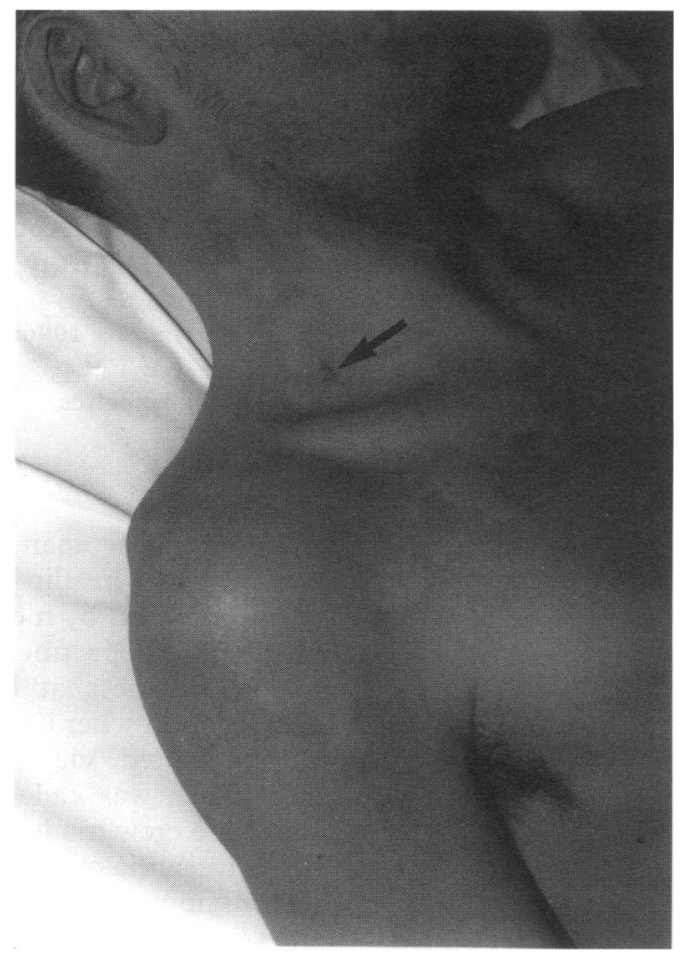

Photograph showing area of central ischaemia with surrounding reactive hyperaemia. Note the injection site in supraclavicular fossa.

chronic intravenous drug abusers, with estimates of approximately 9500 regular users. ${ }^{1}$ Many have been injecting for years and due to the sclerosing effect of the injection have little peripheral venous access. Most, however, have some knowledge of the anatomy of the central veins. When peripheral access is not obtainable, the femoral vein is the route favoured by 